Bull. Korean Math. Soc. 45 (2008), No. 1, pp. 39-44

\title{
SOME RESULTS ON PRECONVEXITY SPACES
}

\author{
Won KeUn Min
}

\begin{abstract}
In this paper, we introduce the concepts of preconvexity neighborhoods, $c$-concave functions. We study some properties for $c$ convex functions, and characterize $c$-convex functions and $c$-concave functions by using the preconvexity neighborhoods.
\end{abstract}

\section{Introduction}

In [1], Guay introduced the concept of preconvexity spaces defined by a binary relation on the power set $P(X)$ of a set $X$ and investigated some properties. He showed that a preconvexity on a set yields a convexity space in the same manner as a proximity [4] yields a topological space.

In this paper, we study some basic properties on preconvexity spaces and cconvex functions. We define the notion of preconvex neighborhood in the same way as a proximity neighborhood is defined by a proximity, and characterize $c$-convex functions and $c$-concave functions by using the preconvex neighborhoods.

Definition 1.1 ([1]). Let $X$ be a nonempty set. A binary relation $\sigma$ on $P(X)$ is called a preconvexity on $X$ if the relation satisfies the following properties; we write $x \sigma A$ for $\{x\} \sigma A$ :

(1) If $A \subseteq B$, then $A \sigma B$.

(2) If $A \sigma B$ and $B=\emptyset$, then $A=\emptyset$.

(3) If $A \sigma B$ and $b \sigma C$ for all $b \in B$, then $A \sigma C$.

(4) If $A \sigma B$ and $x \in A$, then $x \sigma B$.

The pair $(X, \sigma)$ is called a preconvexity space. A convexity is a reflexive and transitive relation. In a preconvexity space $(X, \sigma), G(A)=\{x \in X: x \sigma A\}$ is called the convexity hull of a subset $A$. $A$ is called convex[1] if $G(A)=A$.

Theorem $1.2([1])$. For a preconvexity space $(X, \sigma)$,

(1) $G(\emptyset)=\emptyset$.

Received July 18, 2006; Revised November 22, 2007.

2000 Mathematics Subject Classification. 52A01.

Key words and phrases. preconvexity, preconvex neighborhood, c-concave function.

This work was supported by a grant from Research Institute for Basic Science at Kangwon National University.

(C)2008 The Korean Mathematical Society 
(2) $A \subseteq G(A)$ for all $A \subseteq X$.

(3) If $A \subseteq B$, then $G(A) \subseteq G(B)$.

(4) $G(G(A))=G(A)$ for $A \subseteq X$.

Theorem 1.3 ([1]). If $\sigma$ is a preconvexity on $X$ and $A \subseteq X$, then $G(A)=$ $\cap\{C: G(C)=C$ and $A \subseteq C\}$.

Theorem 1.4 ([1]). Let $\sigma$ be a preconvexity on $X$ and $A, B \subseteq X$. Then

(1) $A \sigma B$ if and only if $A \subseteq G(B)$.

(2) $A \sigma B$ if and only if $G(\bar{A}) \sigma G(B)$.

In [3] Kay and Womble introduced the following definition:

A family $\mathcal{C}$ of subsets of a set $X$ is termed a convexity structure for $X$, and the pair $(X, \mathcal{C})$ is called a convexity space, whenever the following two conditions hold:

(a) $\emptyset$ and $X$ belong to $\mathcal{C}$.

(b) $\cap_{F \in \mathcal{F}} F \in \mathcal{C}$ for each subfamily $\mathcal{F} \subseteq \mathcal{C}$.

If in a preconvexity space $(X, \sigma)$, we take $\mathcal{C}=\{C: C \subseteq X$ and $G(C)=C\}$, then $\mathcal{C}$ is a convexity structure for $X$ called the convexity structure determined [3] by $\sigma$ and $(X, \mathcal{C})$ is a convexity space.

A function $h: P(X) \rightarrow P(X)$ is called a hull operator [3] on $X$ if the following conditions are satisfied:

(1) $h(A) \supseteq A$.

(2) $B \supseteq A$ implies $h(B) \supseteq h(A)$.

(3) $h(\bar{h}(A))=h(A)$.

(4) $h(\emptyset)=\emptyset$.

Let $h$ be a hull operator on $X$. A preconvexity $\sigma$ is said to be associated with the convexity space $(X, h)$ if $x \in h(A)$ implies $x \sigma A$. If in addition, $x \sigma A$ implies $x \in h(A)$, then $\sigma$ is said to be compatible [1] with $(X, h)$.

Definition $1.5([1])$. Let $\left(X, h_{1}\right),\left(Y, h_{2}\right)$ be convexity spaces. A function $f: X \rightarrow Y$ is said to be a convex function on $X$ if for each $A \subseteq X, f\left(h_{1}(A)\right) \subseteq$ $h_{2}(f(A))$. The function $f$ is said to be concave if for each $A \subseteq X, f\left(h_{1}(A)\right) \supseteq$ $h_{2}(f(A))$.

Definition $1.6([1])$. Let $\sigma_{1}, \sigma_{2}$ be two preconvexities on the convexity spaces $\left(X, h_{1}\right)$ and $\left(Y, h_{2}\right)$, respectively. A function $f: X \rightarrow Y$ is said to be $c$ convex if $A \sigma_{1} B$ implies $f(A) \sigma_{2} f(B)$. The function $f$ is a c-isomorphism or convexeomorphism if and only if $f$ is one-to-one, onto and both $f$ and $f^{-1}$ are c-convex.

\section{Main results}

Lemma 2.1. Let $(X, \sigma)$ be a preconvexity space and $A, B \subseteq X$. Then $A \not \phi B$ if and only if there is an element $x \in A$ such that $x \phi B$.

Proof. It is obvious from Theorem 1.4(1). 
Lemma 2.2. Let $(X, \sigma)$ be a preconvexity space. Then for all $A \subseteq X, G(A) \sigma A$.

Proof. From $G(A) \sigma G(A)$ and Definition 1.1(3), it follows the result.

Definition 2.3. Let $(X, \sigma)$ be a preconvexity space and $A \subseteq X . A$ is called a preconvexity neighborhood of $x$, denoted by $x \triangleleft A$, if $x \phi(X-A)$. $A$ is called a preconvexity neighborhood of $B$, denoted by $B \triangleleft A$, if $B \phi(X-A)$.

In a convexity space $\mathcal{C}$, a subset $A$ in $X$ is called a convexity neighborhood of $x$ if there exists a $F \in \mathcal{C}$ such that $x \in X-F \subseteq A$.

Theorem 2.4. Let $(X, \sigma)$ be a preconvexity space and $A \subseteq X$. For $x \in X, A$ is a preconvexity neighborhood of $x$ if and only if it is a convexity neighborhood of $x$ in the convexity space determined by $\sigma$.

Proof. Let $x \triangleleft A$; then by Definition 2.3, $x \triangleleft A$ if and only if $x \phi(X-A)$ if and only if $x \notin G(X-A)$ if and only if $x \in X-G(X-A) \subseteq A$. Thus we get the result because $G(X-A)$ is a convex set.

Theorem 2.5. Let $(X, \sigma)$ be a preconvexity space and $A \subseteq X$. If $A$ is a convexity neighborhood of $B \subseteq X$, then it is a preconvexity neighborhood of $B$ in the convexity space determined by $\sigma$.

Proof. Let $A$ be a convexity neighborhood of $B \subseteq X$; then there is a convex set $F \subseteq X$ such that $B \subseteq X-F \subseteq A$. If $x \sigma(X-A)$ for all $x \in B$, then $B \sigma(X-A)$. Thus $B \subseteq G(X-A) \subseteq G(F)=F$. This is a contradiction. Hence $x \phi(X-A)$ for some $x \in B$, and so $B \phi(X-A)$.

In the following example we can show that the converse in Theorem 2.5 is not always true.

Example 2.6. Let $X=\{a, b, c, d\}$ and define a relation $\sigma$ on $P(X)$ given by $\{d\} \sigma\{a\},\{d\} \sigma\{c\}, X \sigma\{b\}$ and $X \sigma\{a, c, d\}$, and the general condition that $A \sigma B$ if $A \subseteq B$. Then the relation $\sigma$ is a preconvexity on $X$ and $G(\emptyset)=\emptyset, G(X)=X$, $G(\{d\})=\{d\}, G(\{a, d\})=\{a, d\}, G(\{c, d\})=\{c, d\}$. The convexity structure determined by $\sigma$ is $\mathcal{C}=\{X, \emptyset,\{d\},\{a, d\},\{c, d\}\}$. Let $A=\{a, d\}$ and $B=$ $\{a, b, d\}$; since $A=\{a, d\} \phi\{c\}=X-B, B$ is a preconvexity neighborhood of $A$ but it is not a neighborhood of $A$ in the convexity space $\mathcal{C}$.

Theorem 2.7. For a preconvexity space $(X, \sigma)$ if $A \triangleleft B$ and $B \subseteq D$, then $A \triangleleft D$.

Proof. If $A \not D D$, then $A \sigma(X-D)$. Say $B \subseteq D$; then it is $A \nless \nless B$ from the transitive property of preconvexity.

Theorem 2.8. Let $f: X \rightarrow Y$ be a bijective function on two preconvexities $(X, \sigma)$ and $(Y, \mu)$. Then $f$ is c-convex if and only if for $C, D \subseteq Y$ whenever $C \triangleleft D, f^{-1}(C) \triangleleft f^{-1}(D)$. 
Proof. $(\Rightarrow)$ Assume $f^{-1}(C) \not f^{-1}(D)$ for $C, D \subseteq Y$. Then $f^{-1}(C) \sigma(X-$ $\left.f^{-1}(D)\right)$. Since $f$ is surjective and $c$-convex, it follows $C \mu(Y-D)$. Hence $C \not D D$.

$(\Leftarrow)$ Assume $f(A) \mu f(B)$ for $A, B \subseteq X$. Then by Lemma 2.1, there exists an element $y \in f(A)$ such that $y \not f(B)$. Let $f(x)=y$ for some $x \in A$. We can say $y \triangleleft(Y-f(B))$ because of $y \not h f(B)=Y-(Y-f(B))$, and so $f^{-1}(y) \triangleleft(X-B)$. Now we get $x=f^{-1}(y) \phi B$ from definition of the preconvexity neighborhood. Hence $A \not \phi B$.

Theorem 2.9. Let $f: X \rightarrow Y$ be a function on two preconvexity spaces $(X, \sigma)$ and $(Y, \mu)$. Then $f$ is c-convex if and only if $G(f(G(A)))=G(f(A))$ for all $A \subseteq X$.

Proof. $(\Rightarrow)$ Let $f$ be $c$-convex and $y \in G(f(G(A)))$; then $y \mu f(G(A))$. Since $f$ is $c$-convex, from Lemma 2.2, we get $f(G(A)) \mu f(A)$. The transitive property gives $y \mu f(A)$. Thus $G(f(G(A))) \subseteq G(f(A))$. The other inclusion is obvious.

$(\Leftarrow)$ Suppose that $G(f(G(A)))=G(f(A))$ for all $A \subseteq X$. Let $A \sigma B$ for $A, B \subseteq X$; then by Theorem 1.4(1) and hypothesis, we get the following relationship:

$f(A) \subseteq G(f(A)) \subseteq G(f(G(B)))=G(f(B))$.

By Theorem 1.4(1), $f(A) \mu f(B)$.

From Theorem 2.9, we get the following corollary:

Corollary 2.10 (Theorem $12[1]$ ). Let $\sigma_{1}$ and $\sigma_{2}$ be compatible preconvexities on the convexity spaces $\left(X, h_{1}\right)$ and $\left(Y, h_{2}\right)$, respectively. Then $f: X \rightarrow Y$ is convex if and only if $f$ is c-convex.

Theorem 2.11. Let $f: X \rightarrow Y$ be a function on two preconvexity spaces $(X, \sigma)$ and $(Y, \mu)$. Then the following are equivalent:

(1) $f$ is c-convex.

(2) For $A, B \subseteq X$ if $A \sigma B$, then $f(G(A)) \mu G(f(B))$.

(3) $f(G(A)) \mu f(A)$ for $A \subseteq X$.

Proof. (1) $\Rightarrow(2)$ Let $A \sigma B$ for $A, B \subseteq X$; then by Theorem 1.4(2) and Lemma $2.2, G(A) \sigma G(B) \sigma B$. Since $f$ is $c$-convex, $f(G(A)) \mu f(B) \mu G(f(B))$.

(2) $\Rightarrow(3)$ It is obvious from Lemma 2.2 .

(3) $\Rightarrow(1)$ Let $A \sigma B$ for $A, B \subseteq X$; then $A \subseteq G(B)$, and so $f(A) \subseteq f(G(B))$. Hence $f$ is $c$-convex by Definition 1.1(1) and condition (3).

Definition 2.12. Let $\sigma_{1}, \sigma_{2}$ be two preconvexities on the preconvexity spaces $X, Y$, respectively. A function $f: X \rightarrow Y$ is said to be $c$-concave if for $C, D \subseteq Y$ whenever $C \sigma_{2} D, f^{-1}(C) \sigma_{1} f^{-1}(D)$.

Theorem 2.13. Let $f: X \rightarrow Y$ be a bijective function on two preconvexity spaces $(X, \sigma)$ and $(Y, \mu)$. Then $f$ is c-concave if and only if for $A, B \subseteq X$ whenever $A \triangleleft B, f(A) \triangleleft f(B)$. 
Proof. $(\Rightarrow)$ Let $f(A) \Varangle f(B)$ for $A, B \subseteq X$; then $f(A) \mu(Y-f(B))$. Since $f$ is $c$-concave, we have $A \sigma(X-B)$, and so $A \not B$.

$(\Leftarrow)$ Let $f^{-1}(C) \not f f^{-1}(D)$; then there exists an element $x \in f^{-1}(C)$ such that $x \not f f^{-1}(D)$, and let $f(x)=y$. It follows $x \triangleleft\left(X-f^{-1}(D)\right)$, and so $y \triangleleft(Y-D)$. By Lemma 2.1, we get $C \not \mu D$.

Theorem 2.14. Let $\sigma_{1}$ and $\sigma_{2}$ be compatible preconvexities on the convexity spaces $\left(X, h_{1}\right)$ and $\left(Y, h_{2}\right)$, respectively and let $f: X \rightarrow Y$ be a bijective function. Then $f$ is c-concave if and only if it is concave.

Proof. $(\Rightarrow)$ Let $y \in h_{2}(f(A))$ for $A \subseteq X$; then $y \sigma_{2} f(A)$. Since $f$ is $c$-concave and injective, it follows $f^{-1}(y) \sigma_{1} A$. Since $\sigma_{1}$ is compatible with $h_{1}$, we have $f^{-1}(y) \in h_{1}(A)$ and so $y \in f\left(h_{1}(A)\right)$.

$(\Leftarrow)$ Let $f^{-1}(C) q_{1}^{\prime} f^{-1}(D)$ for each $C, D \subseteq Y$; then there exists $x \in f^{-1}(C)$ such that $x f_{1} f^{-1}(D)$, and so $x \notin h_{1}\left(f^{-1}(D)\right)$. Since $f$ is concave and surjective, $f(x) \notin h_{2}\left(f\left(f^{-1}(D)\right)\right)=h_{2}(D)$. Thus we get $f(x) \phi_{2} D$, and so $C \phi_{2} D$.

Corollary 2.15. Let $f: X \rightarrow Y$ be a bijective function on two preconvexity spaces $(X, \sigma)$ and $(Y, \mu)$. Then $f$ is a convexeomorphism if and only if $f$ is $c$-convex and c-concave.

Proof. From Definition 1.6, we get the result.

Theorem 2.16. Let $f: X \rightarrow Y$ be a function on two convexity spaces $\left(X, h_{1}\right)$ and $\left(Y, h_{2}\right)$. Then $f$ is concave if and only if $f\left(h_{1}(A)\right)=h_{2}\left(f\left(h_{1}(A)\right)\right)$ for $A \subseteq X$.

Proof. $(\Rightarrow)$ Let $f$ be a concave function; then $h_{2}\left(f\left(h_{1}(A)\right)\right) \supseteq f\left(h_{1}(A)\right) \supseteq$ $h_{2}\left(f\left(h_{1}(A)\right)\right)$ for $A \subseteq X$. Hence we get $f\left(h_{1}(A)\right)=h_{2}\left(f\left(h_{1}(A)\right)\right)$.

$(\Leftarrow)$ It is obvious.

Theorem 2.17. Let $f: X \rightarrow Y$ be a function on two convexity spaces $\left(X, C_{1}\right)$ and $\left(Y, C_{2}\right)$, then $f$ is concave if and only if for each $A \in C_{1}, f(A) \in C_{2}$.

Proof. It is obvious from Theorem 2.16 .

Acknowledgements. I thank the referee for some useful comments on the paper.

\section{References}

[1] M. D. Guay, Introduction to the theory of convexity-topological spaces, Topology, Vol. II (Proc. Fourth Colloq., Budapest, 1978), pp. 521-545, Colloq. Math. Soc. Janos Bolyai, 23, North-Holland, Amsterdam-New York, 1980.

[2] _ An introduction to preconvexity spaces, Acta Math. Hungar. 105 (2004), no. 3, $241-248$.

[3] D. C. Kay and E. W. Womble,Axiomatic convexity theory and relationships between the Carathéodory, Helly, and Radon numbers, Pacific J. Math. 38 (1971), 471-485.

[4] S. A. Naimpally and B. D. Warrack, Proximity Spaces, Cambridge Tracts in Mathematics and Mathematical Physics, No. 59 Cambridge University Press, London-New York, 1970. 
DePaRtMENT OF MATHEMATICS

KANGWON NaTIONAL UNIVERSITY

Chuncheon 200-701, Korea

E-mail address: wkmin@kangwon.ac.kr 